\title{
4-Peptide Melanoma Vaccine
}

National Cancer Institute

\section{Source}

National Cancer Institute. 4-Peptide Melanoma Vaccine. NCI Thesaurus. Code 691722.

An emulsion of 4 melanoma peptides with potential immunomodulating and antineoplastic activities. Upon vaccination, 4-peptide melanoma vaccine may stimulate an immune response against 4 different melanoma associated antigens. This may lead to a reduction in tumor cell proliferation of cancer cells expressing these antigens. 